CASE REPORT

\title{
Coronary atherosclerotic plaque rupture following thoracic trauma - an uncommon cause of angina and ventricular tachycardia ("torsade de pointes")
}

\author{
Luís Henrique Wolff Gowdak, Márcio Sommer Bittencourt, Carlos Eduardo Rochitte, Luís Alberto Oliveira \\ Dallan, Luiz Antonio Machado César \\ Cardiologia Clínica, Instituto do Coração (InCor), Faculdade de Medicina da Universidade de São Paulo, São Paulo/SP, Brazil.
}

Email: msbittencourt@bol.com.br

Tel.: 551130623124

\section{CASE REPORT}

Blunt thoracic trauma has been previously described as a rare and often missed cause of acute myocardial infarction, ${ }^{1}$ cardiac rupture, ventricular aneurysms, ${ }^{2}$ aortocoronary bypass occlusion, ${ }^{3}$ coronary aneurysms, ${ }^{4}$ angina, ${ }^{5}$ and arrhythmias. $^{6}$

Here, we report the case of a 44-year-old man whose complaints of exertional chest pain and lightheadedness began after suffering a motor-tricycle accident with blunt thoracic trauma 1 month earlier. His past history was unremarkable except for mild hypercholesterolemia; he was overweight and had a familial history of early-onset myocardial infarction.

Immediately after the accident, the patient began to experience chest pain associated with lightheadedness, sweating, and pale skin while walking briskly; the symptoms were relieved by resting and lasted less than five minutes. He sought medical attention in an emergency department from another facility seven days after the accident. Bruises were observed in the epigastrium close to the $12^{\text {th }}$ left costal arch, with tenderness in the left anterior thoracic wall; the physical exam was otherwise normal. No fractures were revealed by a chest X-ray. A 12-lead resting ECG yielded normal results, but CKMB-mass and troponinI levels were slightly elevated. Coronary angiography was performed and revealed a non-obstructive plaque in the left main coronary artery (LM) and 50\% stenosis of the proximal left anterior descending artery (LAD). No obstructions were seen in the circumflex or right coronary artery. Cardiac scintigraphy $\left({ }^{99 \mathrm{~m}} \mathrm{Tc}\right.$ - Sestamibi with dipyridamole) did not indicate any myocardial perfusion defects; a transthoracic echocardiogram revealed preserved left ventricular function and a very small pericardial effusion with no sign of cardiac restriction. The patient was discharged with diagnoses of uncomplicated thoracic trauma and non-critical coronary atherosclerosis, with a referral for medical treatment.

Amlodipine, aspirin, and pravastatin were prescribed, but the patient's symptoms did not improve. Due to the persistence of symptoms, the patient was later seen by a cardiologist, who ordered a treadmill test. During the first

Copyright (c) 2011 CLINICS - This is an Open Access article distributed under the terms of the Creative Commons Attribution Non-Commercial License (http:// creativecommons.org/licenses/by-nc/3.0/) which permits unrestricted noncommercial use, distribution, and reproduction in any medium, provided the original work is properly cited. stage of the Bruce protocol, between the $2^{\text {nd }}$ and $3^{\text {rd }}$ minutes of exercise, the patient developed a non-sustained polymorphic ventricular tachycardia ("torsade de pointes") (Figure 1A), followed by ST-T ischemic changes (Figure 1B).

A high-resolution ECG and cardiac magnetic resonance imaging study were normal, excluding right ventricular dysplasia and myocardial fibrosis due to myocarditis or to an old infarction scar. Previously undiagnosed myocardial ischemia secondary to coronary atherosclerosis was presumed to be the cause for his symptoms, along with exercise-induced ventricular arrhythmia. Surgical myocardial revascularization was therefore advised.

Due to the persistence of symptoms and the high-risk features of the patient's treadmill activity (not only because of the low level of activity associated with ischemic changes but also because of the high-risk arrhythmia associated with the ischemic changes), surgical treatment was chosen as the most appropriate therapy.

The patient underwent an off-pump coronary artery bypass graft with arterial grafts to the LAD and circumflex arteries four weeks after the initial presentation. It should be noted that, during surgery and before the graft was placed, as the surgeon inspected the heart looking for evidence of cardiac trauma, ST-T elevation was observed on the heart monitor. This observation was confirmed by ECG and persisted for $24 \mathrm{~h}$, evolving with new $\mathrm{Q}$ waves in leads II and III and poor progression of R waves in leads V1 through V3, with a striking elevation of CKMB-mass (which peaked at 25 times the normal level $17 \mathrm{~h}$ after surgery). An echocardiogram performed on the second day postoperatively revealed a very small area of hypokinesia in the apex. The patient had an uneventful recovery. An echocardiogram performed at the one-month follow-up was completely normal.

Another treadmill test was performed forty days after surgery; the patient was able to tolerate maximal exercise, and no ECG changes suggestive of myocardial ischemia or arrhythmias were observed. A coronary computed tomography angiogram $(16 \times 0.5-M D C T A$, Aquilion16TM, Toshiba Medical Systems Corporation, Otawara, Japan) with calcium score evaluation revealed a non-calcified atherosclerotic plaque on the proximal segment of the LAD. Inside the plaque, an area of very low density $(<40$ Hounsfield Units) was observed (Figure 2), a finding compatible with a coronary thrombus. Both of the grafts were patent. 

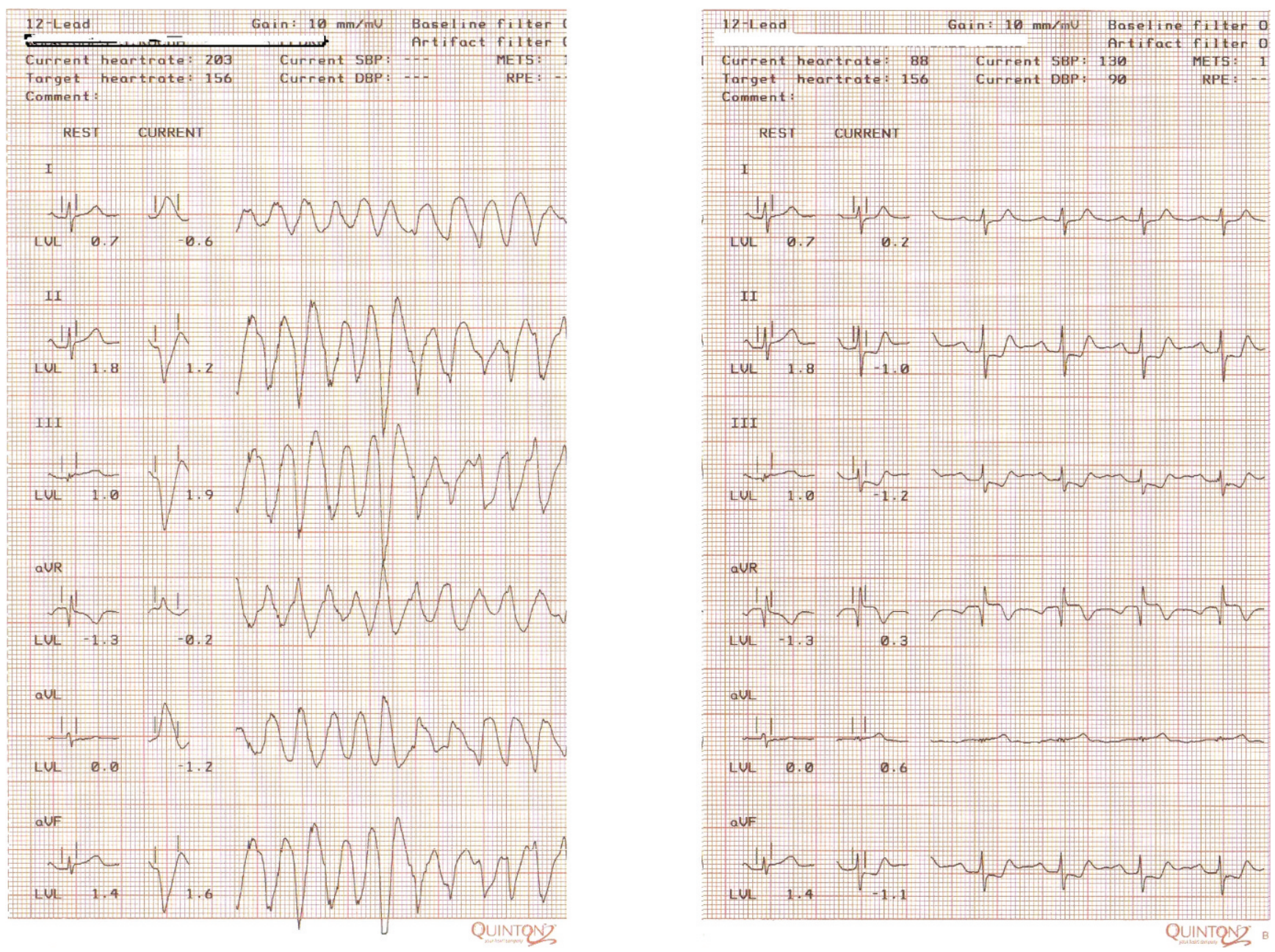

Figure 1 - A treadmill test (Bruce protocol) indicating a non-sustained polymorphic ventricular tachycardia ("torsade de pointes") (Panel A), followed by ST-T ischemic changes (Panel B).

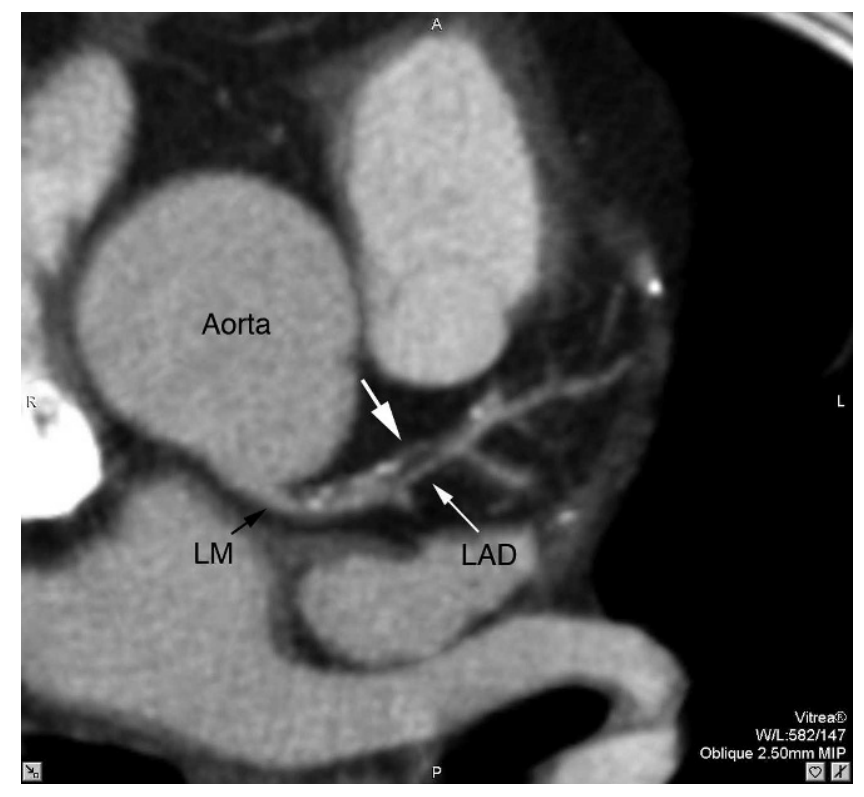

Figure 2 - A coronary-computed tomography angiogram indicating a low-density image in the proximal segment of the LAD that is compatible with a coronary thrombus (white arrows).
Although acute myocardial infarction (AMI) caused by a trauma with laceration of the coronary arteries has been previously described in many case reports, ${ }^{1,7}$ especially in the vicinity of the LAD, most reported cases involved STelevation MI or ventricular aneurysm preceded by a history of trauma, as recently reviewed. ${ }^{8}$ In the present case, myocardial necrosis was detected by serum markers one week after chest trauma, even though the ECG remained normal. Coronary intravascular ultrasound was not recommended to further investigate the extension of the stenotic lesions due to the risk of disrupting the left main plaque. Thus, the initial CK-MB and elevation of troponin might have been caused by direct trauma, whereas the ruptured plaque and thrombus might be responsible for the delayed clinical symptoms.

Notably, there was a striking moment in time relating the thoracic trauma and the onset of anginal symptoms. We believe that a more careful interpretation of the patient's presenting symptoms would have been helpful to identify the cause. Non-invasive imaging revealed a coronary thrombus inside an atherosclerotic plaque, most likely resulting from the chest trauma and leading to exerciseinduced ischemia and malignant ventricular arrhythmia. However, the possibility of post-CABG thrombus formation 
cannot be excluded, as the CT was only performed after surgery.

Another important finding in this case report was the intraoperative myocardial infarction. The most probable etiology of this event was the dislodgement and embolization from the thrombus, which previously rested on the ruptured plaque. Other possible causes of intraoperative myocardial infarction, including hemodynamic instability or surgical technique, are improbable in the present case.

At a follow-up visit one year after the procedure, the patient was doing well and had not experienced the recurrence of any of his symptoms.

\section{ACKNOWLEDGEMENT}

The authors of this manuscript have certified that they are in compliance with the Principles of Ethical Publishing of the International Journal of Cardiology.

\section{REFERENCES}

1. Vasudevan AR, Kabinoff GS, Keltz TN, Gitler B. Blunt chest trauma producing acute myocardial infarction in a rugby player. Lancet. 2003;362:70.

2. Matthew AS, Jefferies JL, McKenzie ED, Ing FF. Traumatic cardiac rupture and left ventricular aneurismal formation in childhood. Am J Cardiol. 2008;101:413-4.

3. Esplugas E, Barthe JE, Sabate J, Fontanillas C. Obstruction of aortocoronary bypass due to blunt chest trauma. Int J Cardiol. 1983;3: 311-4.

4. Lascault G, Komajda M, Drobinski G, Grosgogeat Y. Left coronary artery aneurysm and anteroseptal acute myocardial infarction following blunt chest trauma. Eur Heart J. 1986;7:538-40.

5. Loss DM, MacMillan RM, Maranhao V. Coronary artery obstruction due to blunt chest trauma with residual angina pectoris. Cathet Cardiovasc Diagn. 1983;9:297-301.

6. Sakka SG, Huettemann E, Giebe W, Reinhart K. Late cardiac arrhythmias after blunt chest trauma. Intensive Care Med. 2000;26:792-5.

7. Unterberg C, Buchwald A, Wiegand V. Traumatic thrombosis of the left main coronary artery and myocardial infarction caused by blunt chest trauma. Clin Cardiol. 1989;12:672-4. 